\title{
Manipulating Deformable Linear Objects: Characteristic Features for Vision-Based Detection of Contact State Transitions
}

\author{
Jürgen Acker \\ Dominik Henrich
}

\author{
Embedded Systems and Robotics Lab. (RESY) \\ Faculty of Informatics, Building 48 \\ Kaiserslautern University of Technology, \\ D-67653 Kaiserslautern, Germany
}

E-Mail: [acker, henrich]@informatik.uni-kl.de, http://resy.informatik.uni-kl.de/

\begin{abstract}
This paper deals with the handling of deformable linear objects (DLOs), such as hoses, wires, or leaf springs. It investigates usable features for the visionbased detection of a changing contact situation between a DLO and a rigid polyhedral obstacle and a classification of such contact state transitions. The result is a complete classification of contact state transitions and of the most significant features for each class. This knowledge enables reliable detection of changes in the DLO contact situation, facilitating implementation of sensor-based manipulation skills for all possible contact changes.
\end{abstract}

\section{Introduction}

In most cases, industrial robot systems work only with rigid objects; however manipulation of deformable linear objects (DLOs) such as hoses, wires or leaf springs is desirable, too. For example, the automotive industry must handle DLOs. In particular, the automated installation of cables and hoses in the motor compartment requires a concept for manipulating DLOs. Other application fields are hot wire maintenance or assembly of control cabinets. The main problem in handling DLOs is coping with uncertainties. One particular problem is determining the exact shape of a DLO at the start of a manipulation process, since the shape depends on the preceding manipulation steps. Also, due to manufacturing tolerances, the shape of each individual DLO may differ. During the manipulation, the shape of the DLO changes due to gravity and contact forces. The prediction of such variations with sufficient precision is typically very difficult. The obvious approach is the use of sensors to compensate for these uncertainties.

Some such possibilities are use of a force/torque sensor [6], or implementation of a vision system and force/torque sensor like Nakagaki et al [10]. Both try to solve a special form of the "peg-in-hole" task and thus investigate the solution of clearly specified single tasks. How those special solutions can be applied to more general cases remains unclear.

Much previous research has been performed involving rigid work pieces. The main problem addressed was development of robust and flexible routines (skills) for typical assembly or disassembly tasks. Hasegawa et al. [3] presented the skills "move-to-touch", "rotate-to-level" and "rotate-to-insert" for handling rigid objects. Those skills encapsulate the programming-intensive sensor data processing and can be used for solving complex assembly or disassembly problems, like the disassembly of a valve [3]. Later research addressed the problem of finding a universal set of manipulation skills. Morrow and Khosla proposed a taxonomy to develop manipulation task primitives for composing sets of robot skills likely to cover a given domain [8] and [9].

For deformable linear objects, a similar approach based on contact states was introduced by Henrich et al. [4]. As this was the basis for the work presented here, the next section describes this approach in more detail. Remde [11] analyzed the possible transitions between contact states and implemented a force-based recognition of those contact state transitions in [12] and [13]. This method was implemented in [14], using assembly of a leaf spring as an example of use. The results showed that some transitions between contact states are hard to recognize with force sensor data, but the additional use of other types of sensors may solve this problem. We propose vision sensors such as a CCD camera as promising solutions. To verify this, vision-based recognition is investigated independently. A first attempt at vision-based recognition of contact state transitions was made in [1], based on a qualitative description of the progress of four features, but is not complete. Further, the relevance of the proposed features as the basis for the recognition is not clear and the image processing depends on some strong assumptions, which should be weakened.

Here, we propose to find the features most characteristic of transitions that are widely independent of the underlying low-level algorithms for image processing or object representation. Another objective is assigning the different transitions to classes and generating sensor driven routines for detecting such transitions.

In Section 2, we first describe previous work and our working assumptions. Section 3 describes our basic approach for visual recognition of contact state transitions. In each of the following sections (Section 4 through 6), we introduce one class of transitions and describe the characteristic features for each class. In 
Section 7, we summarize the results and give an overview on the future work.

\section{Previous Work}

The work described here, depends heavily on the contact states for DLOs introduced in [4] and [11], thus, the following is a summary of that research. Since a polyhedral environment is assumed in [4], only vertices, edges, and faces exist as geometric primitives. The DLO is modeled by two vertices with an edge between them. The resulting contact states are shown in Figure 1.

A contact is considered to be stable if and only if a small movement in any direction does not change the contact state. (Even if the position of the contact point or contact line changes.) This requires a contact force, since otherwise only instable contacts exist. Only stable contact states can be established and released in a reliable manner. The instable contact states behave uncontrollably as described below.

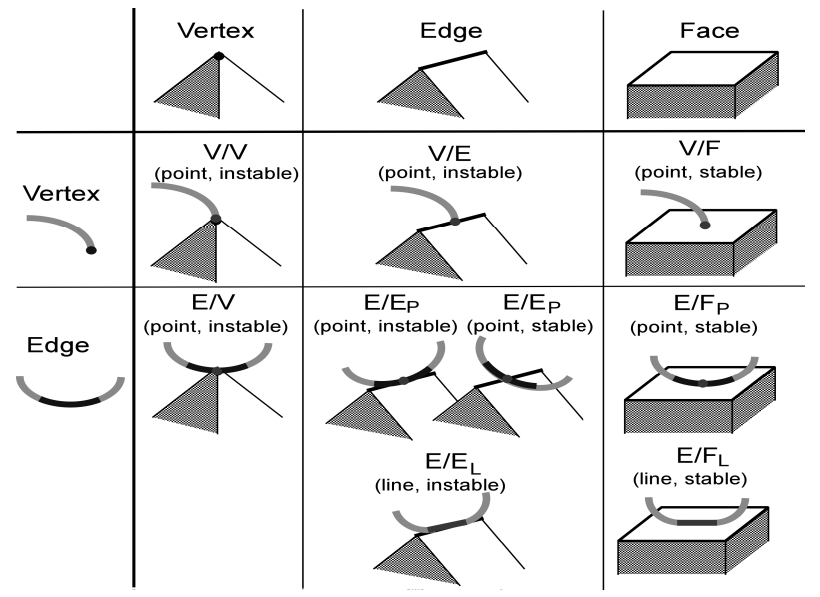

Figure 1: $\quad$ Enumeration of contact states ${ }^{1}$.

Before the transitions between contact states are examined, we first further investigate the effects of the required contact force. This contact force presses the DLO against the obstacle, causing a deformation. For the stable $\mathrm{E} / \mathrm{F}_{\text {Point }}$ and the instable $\mathrm{E} / \mathrm{E}_{\text {Point }}$ contact states, the force flattens the DLO at the contact point, resulting in a short line contact instead of real point contact. Therefore, both contacts behave like their corresponding line contacts, so we will only further discuss the stable $\mathrm{E} / \mathrm{F}_{\mathrm{Line}}$ and stable $\mathrm{E} / \mathrm{E}_{\text {Point }}$ contact states. The instable $\mathrm{E} / \mathrm{E}_{\text {Line }}$ contact state is also regarded. Because of the obstacle's geometric properties, the stable $\mathrm{E} / \mathrm{E}_{\text {Point }}$ and the $\mathrm{E} / \mathrm{V}$ contact remain point contacts.

After defining contact states, we must now consider the possible transitions between them. A contact state transition is a change from one contact state to another without passing any intermediate contact states. For the handling of DLOs, the stable states are most interesting,

\footnotetext{
${ }^{1}$ From $[4,11]$ but the instable E/E point contact has been added.
}

since the instable ones change spontaneously to a stable state and such spontaneous transitions cannot be controlled. Since the spontaneous transitions lead to stable contact states, the instable ones may still occur as transient states after an initiated state transition leads to an instable state. The resulting contact states and state transitions are shown in Figure 2, with regard only to single contacts.

In addition to the assumptions mentioned above, such as a polyhedral environment and contact forces, we make some assumptions for the vision system. First, we restrict ourselves presently to elastic DLOs with emphasis on low elastic deformation (E+, E- classes in [4]). Highly elastic objects may oscillate after acceleration, but we assume here that objects will either not oscillate or that an active damping [15] took place. After an initial acceleration, the acceleration of the gripper should be zero (linear motion) until the contact state transition takes. Further, the camera is placed at the optimal observation point for each transition and the environment is assumed to be static, so only the robot and the manipulated object move, and the other obstacles remain unchanged during the manipulation process.

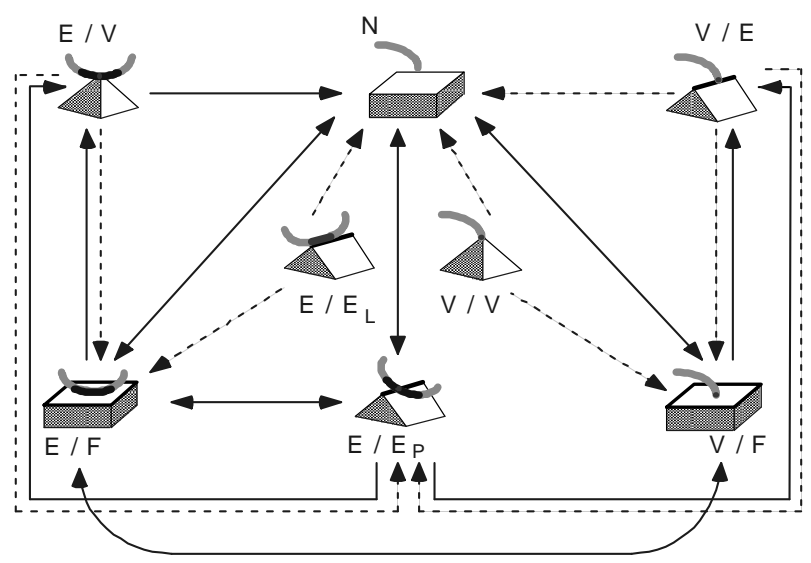

Figure 2: $\quad$ State transition graph, solid edges indicate initiated and dashed indicate spontaneous transitions ${ }^{2}$.

\section{Basic Approach}

One method for vision-based detection of contact state transitions is the use of an object recognition system and generation of a 3D model of the DLO and the environment. However, since computer vision is still much inferior to human vision, the development of a system for recognizing DLOs and calculation of a 3D model is still an expensive task, especially the calibration of the camera(s). Fortunately, the introduced approach does not require a precise model for DLOs due to abstracing from geometric details.

In [13], a force/torque sensor was used to detect contact state transitions instead of a vision system. Since

\footnotetext{
${ }^{2}$ From [11] but the instable states E/E and V/V have been added.
} 
the force/torque sensor is unable to sense the environment and the method described here does not use a 3D model, our vision-based system may not need such a 3D model . The internal forces measured with the force/torque sensor deform the work-piece, resulting in observable deformation. Indeed, [7] describes an approach for fusing vision and force sensor data, whereby the vision sensor is used as an additional force/torque sensor by calculating the forces based on object deformation. This means identifying vertices, edges or faces is not necessary for detecting contact state transitions.

For this reason and because we assume a static environment, we can use the work piece motion to segment background and foreground [5]. Our approach is basically a flow-based one, since we always regard a sequence of images, but we do not restrict ourselves to any special technique such as optical flow. More concretely, we use a stationary gray-value camera to acquire images and remove the static environment from the image by calculating difference images. In the next step, binary images are calculated by applying a threshold value to the difference images. In the resulting binary images, only the work-piece and the robot remain (Figure 3). The remaining sequence of threshold-applied difference images (hereafter: difference images) should contain sufficient information for successful vision-based detection of contact state transitions.

But now we must answer the question of recognizing contact state transitions in such a sequence of difference images. Since we do not want to restrict ourselves to any specific algorithm, our objective is the identification of general features that are significant for detecting state transitions. The development of algorithms for detecting/extracting those features from the image sequence is part of future work.

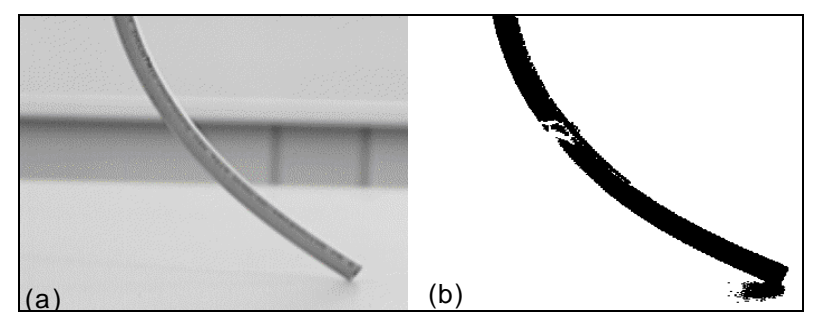

Figure 3: The original image taken by a grey-value camera (a) and the resulting binary image (b).

Any algorithms developed must fulfill some requirements, an important one being reliability. A reliable algorithm must detect every transition and the contact states after the transition must be stable. In particular, we need a contact force to achieve a stable contact. So, for rigid objects the transitions must be detected as fast as possible; otherwise the object or environment may suffer damage. In contrast, with a DLO, the transition must not be detected so quickly or an instable contact may be the result.

Instead of looking for features for every single contact state transition, similar transitions are grouped together in classes, with each class handled by one skill and its inversion. Since force-based transition detection uses one skill for every single transition, the following classification mainly deals with vision-based methods.

The next sections describe three classes of contact state transitions and the features for detecting them. The classes are similar but not identical to the three skills used by Hasegawa [3] for handling rigid objects, since here we regard deformable objects based on the state transitions shown in Figure 2. While no set of transitions corresponds to his "rotate-to-insert" skill and the last class described here only applies to deformable objects, his "move-totouch" and "rotate-to-level" skills are almost identical to the first and second class described below.

\section{Establishing and Releasing Contact}

The move-to-touch skill is useful for handling deformable objects and is represented in Figure 2 by all edges from $\mathrm{N}$ to any other state. In contrast to Hasegawa [3], we also introduce a "move-to-detach" skill. This skill is the inversion of the move-to-touch skill and is represented by any initiated transitions to N. Both skills belong to the establishing- and releasing-contact class.

Since we always assume a contact force for any existing contact, only motions leading to such a contact force are allowed. Two examples for allowed motions are given in Figure $4 \mathrm{~b}$ and $4 \mathrm{~d}$. The motions $4 \mathrm{a}$ and $4 \mathrm{c}$ are examples of establishing a contact without deformation of the work piece. Such motions are not allowed, since they do not lead to a contact force. The rest of the paper concentrates on translatory motions like $4 \mathrm{~b}$, but the features described work also with rotatory motions like $4 d$.

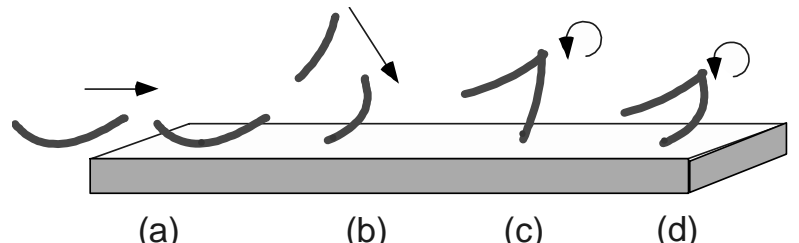

Figure 4: $\quad$ Establishing contact via translatory motions $(a, b)$ or rotatory motions $(c, d)$ while deforming $(b, d)$ or not deforming $(\mathrm{a}, \mathrm{c})$ the work piece.

The first feature examined for vision-based transition detection is of course deformation. As long as the DLO is not in contact with any obstacle (but the gripper), it may deform due to gravity. Establishing a stable contact leads to a deformation caused by the contact force. This deformation may be measured by observing the curvature of the DLO. The main characteristic of an established contact is that at least a part of the DLO stops moving in at least one direction, while other parts continue their motion. This can be seen in Figure 5, where two sequences of difference images are shown. Every sequence consists of four binary images; the brighter the DLO, the older the corresponding difference image. In 
both sequences, the DLO is moved in the $v$-direction until it hits an obstacle (box). In the left sequence, the tip of the DLO stops moving in the $v$-direction and starts moving in the negative $u$-direction. If due to friction or the DLO's shape it does not move in the $u$-direction, the $v$-direction motion will stop at the contact point, since the obstacle prevents further motion. Either stoppage of motion in the $v$-direction or additional motion in the $u$-direction will always occur. The right-hand sequence also shows this effect, whereby the marked part stops moving in the $v$ direction. Thus, the common feature is that part of the DLO stops moving in one direction as it hits an obstacle. This is the characteristic feature for all transitions from $\mathrm{N}$ to any other contact state, including all instable ones.

This loss of motion may be detected by comparing successive difference images. The result of such a comparison of the last two images is shown at the bottom line of Figure 5. The later an image is taken, the more the DLO has moved in the motion direction until the DLO hits the obstacle. We can then distinguish up to three areas $a^{-}, a^{+}$and $a^{0}$. In $a^{+}$the DLO continues moving but in $a^{0}$ it remains at the same place, whereas in area $a^{-}$, the DLO even moves backwards. The existence of an area $a^{0}$ is equivalent to a stop in motion; thus, it appears in both examples.

The reverse transitions from any state to $\mathrm{N}$ can be detected in the same way, but the characteristics are inverted. This means that we start with an $a^{0}$ area and after the contact state transition, only the $a^{+}$area remains.

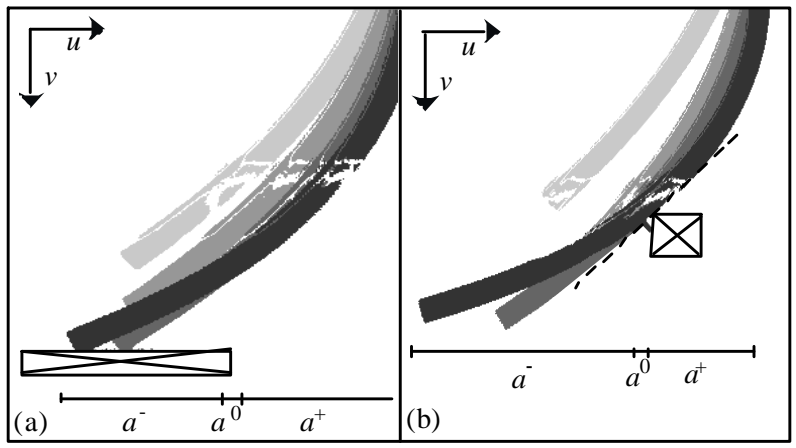

Figure 5: Sequence of difference images for the transition $\mathrm{N} \rightarrow \mathrm{V} / \mathrm{F}$ (a) and for $\mathrm{N} \rightarrow \mathrm{E} / \mathrm{E}_{\mathrm{P}}(\mathrm{b})$; for better orientation the obstacle is also shown.

More difficult than detection of contact is the identification of the resulting contact state. Since our basic approach uses difference images, the obstacles are invisible in the binary images. Additionally, it cannot be decided whether the obstacle is an edge or a face. The sequence in Figure $5 b$ shows an $\mathrm{N} \rightarrow \mathrm{E} / \mathrm{E}_{\mathrm{P}}$ transition. An obstacle represented by the dotted line would produce the same sequence of images, but the transitions would be $\mathrm{N} \rightarrow \mathrm{E} / \mathrm{F}$. Since we cannot sense what geometric primitive the DLO is in contact with, we can only try to decide whether the edge or the vertex of the DLO is in contact. However, even this task is difficult since the deformation due to stopped motion may not appear at the tip even for a vertex contact (Figure 5a). But since the tip of the DLO stops moving in the motion direction for any vertex contact, this enables one to distinguish DLO vertex- from edge-contacts.

\section{$5 \quad$ Stable State Transitions}

The second class contains all immediate transitions from any stable contact state other than $\mathrm{N}$ to any other stable contact state other than $\mathrm{N}$, like $\mathrm{V} / \mathrm{F} \rightarrow \mathrm{E} / \mathrm{F}$. This class corresponds to Hasegawa's rotate-to-level skill for rigid objects, because his skill and this class share the main characteristic, namely the change from a point to a line contact. But in contrast to the case of rigid objects, where this can only be done by a rotatory motion, in DLOs both translatory and rotatory motion can change a point to a line contact. This class also contains the reverse transitions from line to point contacts.

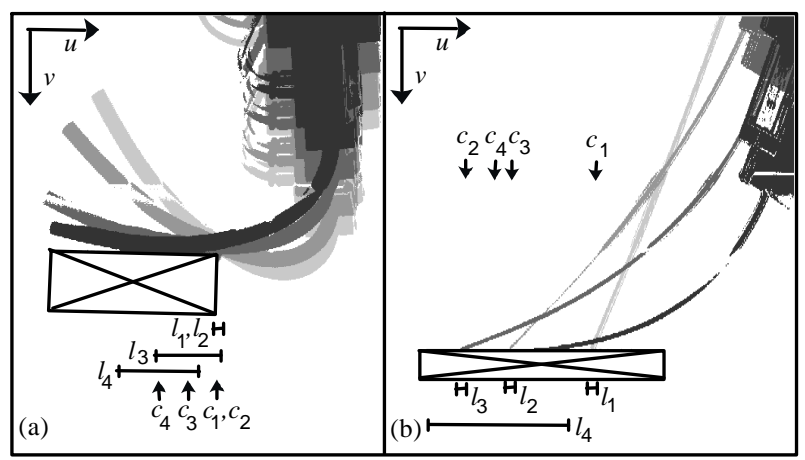

Figure 6: $\quad$ Sequence of difference images $i=1, \ldots, 4$ for the transition $\mathrm{E} / \mathrm{E}_{\mathrm{P}} \rightarrow \mathrm{E} / \mathrm{F}$ (a) and $\mathrm{V} / \mathrm{F} \rightarrow \mathrm{E} / \mathrm{F}$ (b) with contact length $l_{\mathrm{i}}$ and centre of contact $c_{\mathrm{i}}$.

The deformation change depends on the motion. For example, the transition $\mathrm{V} / \mathrm{F} \rightarrow \mathrm{E} / \mathrm{F}$ can be made without further (visible) deformation of the work piece by using the contact point as rotation centre. Translatory motions must deform the work piece, for this reason a rotate-tolevel motion cannot be substituted with a translatory motion for rigid objects.

The deformation offers a way to recognize such contact state changes, assuming a translatory motion. But the bending of the DLO may increase (Figure 6b) or decrease (Figure 6a), depending on the specific geometric situation. Although the shape changes heavily, it is difficult to determine the transition point, so additional features such as the length of the contact should be regarded. It is obvious that a point contact is smaller in length than a line contact, so this can easily be used as a feature. The examples in Figure 6 show two transitions from a point to a line contact, whereby the contact length $l_{\mathrm{i}}$ is drawn for every image. Another feature is the motion of the center of contact $c_{\mathrm{i}}$. This centre is unchanged as long as the work-piece is in the $\mathrm{E} / \mathrm{E}_{\mathrm{P}}$ contact state (Figure 6a) but after the transition to $\mathrm{E} / \mathrm{F}$, it starts moving. In the second sequence (Figure 6b), the centre first moves to the left while the work piece is in $\mathrm{V} / \mathrm{F}$ and after reaching $\mathrm{E} / \mathrm{F}$ it moves back $\left(c_{4}\right)$. The reverse transitions 
$\mathrm{E} / \mathrm{F} \rightarrow \mathrm{V} / \mathrm{F}$ and $\mathrm{E} / \mathrm{F} \rightarrow \mathrm{E} / \mathrm{E}_{\mathrm{P}}$ can be detected in the same way, but the characteristics must be inverted.

The deformation and the length of contact are quantitative features, since the features do not change their sign at the contact point. In contrary, the contact point motion is more a qualitative feature because the centre of contact either begins moving and stops after the transition or changes the direction of its motion. Thus, this feature is expected to be more resistant to noise, making it more reliable than others. The contact state validation here is as difficult as for the establishing and releasing contact class but as the transitions can be fully controlled, an additional validation is not really necessary.

\section{Spontaneous Transitions}

All transitions in the transition graph (Figure 2) marked spontaneous and those leading from stable to instable states still remain to be classified.

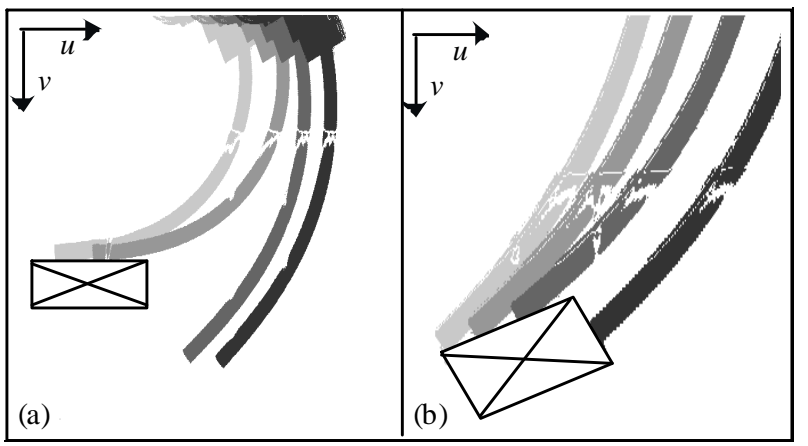

Figure 7: $\quad$ Sequences of difference images for a transition $\mathrm{V} / \mathrm{F} \rightarrow \mathrm{V} / \mathrm{E} \rightarrow \mathrm{N}$ (a) and $\mathrm{V} / \mathrm{F}_{1} \rightarrow \mathrm{V} / \mathrm{E} \rightarrow \mathrm{V} / \mathrm{F}_{2}$ (b).

Those transitions ending in an instable state cannot be detected with the features discussed in the last section because the contact would remain a point $(\mathrm{V} / \mathrm{F} \rightarrow \mathrm{V} / \mathrm{E})$ or line contact $\left(\mathrm{E} / \mathrm{F} \rightarrow \mathrm{E} / \mathrm{E}_{\mathrm{L}}\right)$. Figure $7 \mathrm{~b}$ shows such a transition $(\mathrm{V} / \mathrm{F} \rightarrow \mathrm{V} / \mathrm{E})$ and the following spontaneous transition $(\mathrm{V} / \mathrm{E} \rightarrow \mathrm{V} / \mathrm{F})$. Humans can recognize the $\mathrm{V} / \mathrm{F} \rightarrow \mathrm{V} / \mathrm{E}$ transition in the original sequence of grayvalue images. However, in the sequence of difference images even a human fails to detect this transition, due to the need for edge-recognition capability. Fortunately, detection of this transition is not even necessary, since (due to instability of V/E) another recognizable transition takes place. Here, this spontaneous transition leads to the stable state $\mathrm{V} / \mathrm{F}$, a transition recognizable even in sequences of difference images.

In general, several stable states can be reached from an instable one. Which one is actually reached depends on several conditions; an exact analysis of the transition conditions can be found in [7]. Whatever stable contact state results, there is always a spontaneous transition after reaching an instable contact state leading to a stable one. Thus, the third class does not contain single transitions but sequences of transitions. Such transition sequences consist of an initiated transition to an instable state and a spontaneous transition to a stable state. Since the spontaneous part is visible in the sequence of difference images, the sequence is recognizable even if the first part cannot detected by our approach. Contrary to initiated transitions, spontaneous transitions are one-way transitions, so only one skill is needed for this class. Further, since such spontaneous transitions lead often to state $\mathrm{N}$, this class would intersect with the establishing and releasing contact class. Thus, the restriction to "initiated transitions" for the establishing and releasing contact class is made.

First, we assume the resulting state is not N. One way to detect such a transition is observing the contact point motion. If the DLO is dragged over an obstacle, the contact point always follows the surface of the obstacle as long as it is in contact. Since only a polyhedral environment is considered, the contact point always moves along a straight line (see Figure 7). A transition from any stable contact state to another contact state via an instable one causes a discontinuity. In Figure $7 b$, the face before and after the $\mathrm{V} / \mathrm{E}$ contact state must have different normal vectors, otherwise both faces are identical and there is no transition. In general, the orientation of the geometric primitives before and after the transition sequence must differ. Since the contact point must follow the surface, this change of orientation means the contact point now follows a straight line with a different direction vector than before.

In the second case, (Figure 7a), the contact is released after the spontaneous transition, but this can be sensed with the features discussed in Section 4 or by observing the deformation of the DLO. The characteristic of spontaneous transitions is the release of stress. This makes such a transition uncontrollable but also reduces bending of the work piece. The motion before the spontaneous transition may deform the DLO and may even reduce bending, but the transition itself at least causes an additional reduction, so there is always a discontinuity while observing the change of deformation. In addition, usually straightening occurs rather quickly and initiated transitions cause continual (un)bending, so fast changes are typical for spontaneous transitions.

A state validation is again difficult but at least it is possible to distinguish the transition leading to $\mathrm{N}$ from those leading to any other stable state. This is possible by examining features used to detect release of a contact.

\section{Conclusions}

We identified three classes in particular: one for establishing and releasing contact, one for changing from one stable contact state to any other state but $\mathrm{N}$ and one for the spontaneous transitions. For each class, some of what we believe to be characteristic features for visionbased detection are given. The listed features allow for recognition all transitions in Figure 2 but the transitions from any stable state (except $\mathrm{N}$ ) to any instable state. For recognizing such transitions, the recognition of the environment is necessary. However, the instability of 
such contacts results in a spontaneous transition leading to a stable contact state. Since the detection of such spontaneous transitions is possible, recognition of the environment is not needed. But one disadvantage remains, since without recognition of the environment, we cannot identify the contact state after the transition. Only a weak estimation is possible without further activities such as probing or exploration motions. The advantage of our approach is the simplicity; we foresee neither the need for calibrated cameras nor for a large, time consuming system for object recognition.

Remaining steps include development of some lowlevel image processing algorithms for extracting the described features from the sequence of difference respective binary images. Our focus here is again on simple, reliable and fast algorithms. The algorithm will be based on comparison of successive difference images like those shown in Figures 5 through 7. The successive difference images will be merged as in these figures, but as every image is to be used, the sequence becomes much smoother than in the figures shown. The resulting image is a good basis for calculating optical flow [15], and the optical flow field is expected to be sufficient for detecting the described features.

It is obvious that for the recognition of any transition, the camera must be able to observe it. Therefore, the placement of the camera is another topic for future work. The image sequences shown in Figures 5 through 7 are taken from a side view. Indeed, an angle of 90 degrees between the camera axis and the plane spanned by the movement vector of the DLO and the normal vector of the obstacle's surface was used. But as we expect a fair amount of angular independence, an angle of perhaps 45 degrees should also work. However, there is a big difference between this side view and a top view. Since even for a human recognizing the contact state transitions based on a top view is very difficult, we do not expect any algorithm based on the features described above to function in this case.

In general, more thorough investigation of where we can or must place the camera is needed. In this context, we will also investigate the required lightning conditions.

\section{References}

[1] Abegg F., Henrich D. and Wörn H.: "Manipulating deformable linear objects - Vision-based recognition of contact state transitions". In: Proc. of the $9^{\text {th }}$ Int. Conf. on Advanced Robotics, Tokyo, Japan 1999

[2] Davis J. W.: "Recognizing Movement using Motion Histograms" in: MIT Technical Report \#487, 1999

[3] Hasegawa T., Suehiro T., and Takase K.: “A modelbased manipulation system with skill-based execution". In: IEEE Transactions on Robotics and Automation, vol. 8, No. 5, pp. 535-544, October 1992

[4] Henrich D., Ogasawara T., Wörn H.:"Manipulating deformable linear objects: Contact states and point contacts". In: Proc. IEEE International Symposium on Assembly and Task Planning, Porto, Portugal, 1999.

[5] Jain R, Martin W.N. and Aggarwal J.K.: "Segmentation Through the Detection of Change Due to Motion“ In: Computer Graphics Image Processing No. 1 September 1979, pp. 13-34.

[6] Kraus W., McCarragher B. J.: "Case studies in the manipulation of flexible parts using a hybrid position/force approach". In: Proc. 1997 Int. Conf. on Robotics and Automation, vol.. 1, pp. 367372, Albuquerque, USA, April, 1997.

[7] Luo Y. and Nelson B.J.: "Fusing Force and Vision Feedback for Manipulating Deformable Objects", Journal of Robotic Systems 18(3), pp. 103-117 2001

[8] Morrow J. D., Khosla P. K.: "Manipulation task primitives for composing robot skills". In: Proc. 1997 IEEE Int. Conf. on Robotics and Automation, pp. 3354-3359, Albuquerque, USA, April 1997.

[9] Morrow J. D., Nelson B. J., Khosla P. K.: "Vision and force driven sensorimotor primitives for robotic assembly skills". In: Proc. 1995 IEEE/RSJ Int. Conf. on Intelligent Robots and System, Pittsburgh, Pennsylvania, USA, August 1995.

[10] Nakagaki H., et al.: "Study of deformation and insertion tasks of a flexible wire". In: Proc. 1997 Int. Conf. on Robotics and Automation, vol. 3, pp. $2397-$ 2402, Albuquerque, USA, April 1997.

[11]Remde A., Henrich D., and Wörn H.: "Manipulating deformable linear objects: Contact state transitions and transition conditions". In: IEEE/RSJ Int. Conf. on Intelligent Robots and Systems, Kyongju, Korea, October 1999.

[12] Remde A., Pfaffenberger E., and Wörn H.: "Manipulating deformable linear objects: Force based detection of contact state transitions". In: IEEE/RSJ Int. Conf. on Intelligent Robots and Systems, Takamatsu, Japan, October/November 2000.

[13] Schlechter A. and Henrich D.: "Manipulating Deformable Linear Objects: Characteristics in Force Signals for Detecting Contact State Transitions" In: Proc. of $10^{\text {th }}$ Int. Conf. on Advanced Robotics Budapest, 22-25. August 2001.

[14] Schlechter A and Henrich D. "Manipulating Deformable Linear Objects: Programming using Different Manipulation Skills" In: VDI Bericht 1679 Tagungshandbuch zur Robotik 2002 June 19-20, Ludwigsburg Germany 2002

[15] Schlechter A. and Henrich D.: "Manipulating Deformable Linear Objects: Manipulation Skill for Active Damping of Oscillations“ In: IEEE/RSJ Int. Conf. on Intelligent Robots and Systems, Lausanne, Switzerland 2002. 